\title{
Enhanced self-phase modulation in silicon nanowires integrated with 2D graphene oxide films
}

\author{
Yuning Zhang ${ }^{1}$, Jiayang Wu ${ }^{1}$, Yunyi Yang ${ }^{1,2}$, Yang Qu ${ }^{1}$, Linnan Jia ${ }^{1}$, Tania Moein ${ }^{1}$, \\ Baohua Jia ${ }^{1,2}$, and David J. Moss ${ }^{*}$ \\ 1. Centre for Micro-Photonics, Swinburne University of Technology, Hawthorn, Victoria 3122, Australia. \\ 2. Centre for Translational Atomaterials, Swinburne University of Technology, Hawthorn, Victoria 3122, Australia.
}

\begin{abstract}
Two-dimensional layered graphene oxide (GO) films are integrated with silicon-oninsulator nanowires to experimentally demonstrate enhanced self-phase modulation, achieving high broadening factor of up to 4.14 for a device patterned with 0.4-mm-long, 10 layers of GO.
\end{abstract}

\section{Introduction}

All-optical integrated photonic devices, with compact footprint, low power consumption, high stability and the potential to significantly reduce cost by mass production, are critical for high-speed signal generation and processing in optical communication systems [1]. Although silicon has been a leading platform for integrated photonic devices, its strong two-photon absorption (TPA) at near-infrared wavelengths that induces undesired free carrier absorption (FCA) and dispersion (FCD) poses a fundamental limitation for the nonlinear performance of silicon photonic devices in the telecommunications band $[1,2]$. This has motivated on-chip integration of new platforms $[3,4]$ as well as advanced nonlinear materials such as two-dimensional materials to overcome the limitation of silicon $[5,6]$.

Among the various 2D materials, GO has received increasing interest due to its ease of preparation as well as the tunability of its material properties [7-10]. Recently, we reported GO films with a giant Kerr nonlinear response about 4 orders of magnitude higher than that of silicon and demonstrated enhanced four-wave mixing (FWM) in doped silica waveguides integrated with GO films [8]. In this paper, [11] we further demonstrate enhanced self-phase modulation (SPM) in silicon-on-insulator (SOI) nanowires integrated with 2D layered GO films. We perform SPM measurements using picosecond optical pulses for the SOI nanowires integrated with 2.2-mm-long, 1-3 layers of GO and 0.4-mm-long, 5-20 layers of GO, respectively. Owing to the strong light-matter interaction between the SOI nanowires and the highly nonlinear GO film, we observe a significant spectral broadening for the GO-coated SOI nanowires as compared with the uncoated SOI nanowires, achieving a high broadening factor (BF) of up to 3.63 for an SOI nanowire with 2.2-mm-long, 2 layers of GO and 4.14 for a device with 0.4-mm-long, 10 layers of GO. These results confirm the effectiveness of introducing 2D layered GO films into integrated silicon photonic devices to improve the performance of nonlinear optical processes.

\section{Device fabrication and characterization}

Figure 1(a) shows a schematic of an SOI nanowire waveguide integrated with a GO film. The SOI nanowire with a cross section of $500 \mathrm{~nm} \times 220 \mathrm{~nm}$ was fabricated on an SOI wafer using 248-nm deep ultraviolet photolithography followed by an inductively coupled plasma etching process. Inverse taper couplers were employed to couple light into and out of the devices with lensed fibers. A $1.5-\mu \mathrm{m}$ thick silica layer was deposited by plasma enhanced chemical vapor deposition as an upper cladding layer. Finally, windows with two different lengths of $0.4 \mathrm{~mm}$ and $2.2 \mathrm{~mm}$ were opened down to the buried oxide layer to allow the introduction of GO films. Figure 1(b) shows a microscope image of a fabricated SOI chip with a 0.4-mm-long opened window. The GO film was introduced onto the silicon chip by a solution-based method that yielded transfer-free, layer-by-layer GO film deposition [6, 7]. This method allows conformally coating GO films onto the silicon waveguide, as shown by the scanning electron micrograph (SEM) in Fig. 1(c) and the Raman spectra in Fig. 1(d).

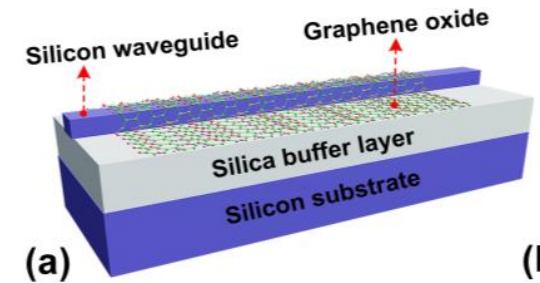

(b)
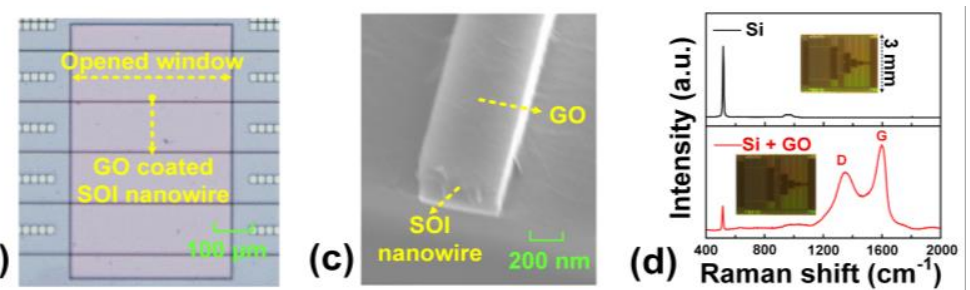

Fig. 1. (a) Schematic illustration of a GO-coated SOI nanowires. (b) Microscope image of a fabricated SOI chip with a 0.4-mm-long opened window. (c) Scanning electron microscopy (SEM) image of a SOI nanowire conformally coated with 1 layer of GO. (d) Raman spectra of an integrated SOI chip without GO and with 2 layers of GO. Insets show the corresponding microscope images. 
We measured the insertion loss using a low-power $(0 \mathrm{dBm})$ transverse electric (TE) polarized continuous-wave (CW) light. The propagation loss of the bare waveguide and the waveguide with a monolayer of GO was $\sim 0.42$ $\mathrm{dB} / \mathrm{mm}$ and $\sim 2.05 \mathrm{~dB} / \mathrm{mm}$, respectively, corresponding to an excess propagation loss of $\sim 1.63 \mathrm{~dB} / \mathrm{mm}$ induced by the GO film. This is over 1 order of magnitude lower than that of silicon waveguides coated with graphene [5].

\section{SPM in GO-silicon hybrid waveguides}

Figures 2(a) and (b) show normalized optical spectra of the picosecond optical pulses (pulse width: $\sim 3.7 \mathrm{ps,} \mathrm{repetition}$ rate: $\sim 60 \mathrm{MHz}$ ) after going through the hybrid waveguides with 2.2-mm-long, 1-3 layers of GO and 0.4-mm-long, 5-20 layers of GO, respectively, together with those of the input pulses and the pulses after passing the bare SOI nanowire. For comparison, we kept the same input pulse energy of $51.5 \mathrm{pJ}$. As compared with the bare SOI waveguide, there are obviously broadened spectra for all the hybrid waveguides. The SOI nanowires with a shorter GO coating length and higher film thicknesses even show more significant spectral broadening than the SOI nanowires with a longer GO coating length and lower film thicknesses. The optical spectra measured with different input pulse energies for the waveguides with 2 layers and 10 layers of GO are shown in Figs. 2(c) and (d), respectively.

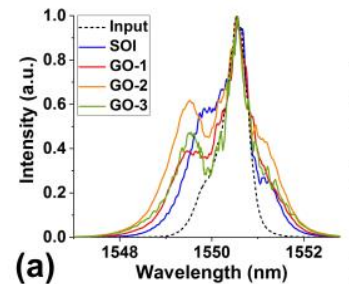

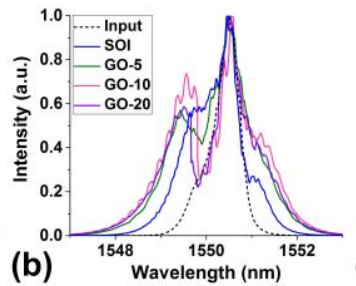

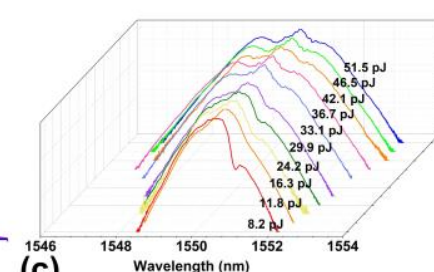

(c)

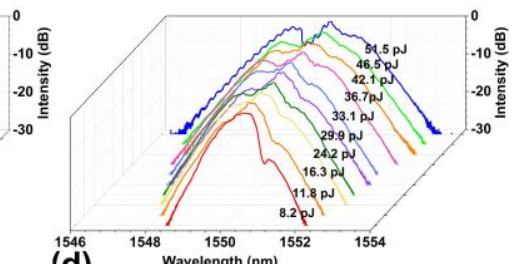

(d)

Fig. 2. (a-b) Normalized optical spectra of the picosecond optical pulses before and after going through the bare and hybrid waveguides integrated with 2.2-mm-long , 1-3 layers of GO and 0.4-mm-long ,5-20 layers of GO, respectively. (c-d) Optical spectra measured with different input pulse energies for the waveguides with 2.2-mm-long, 2 layers of GO and 0.4-mm-long, 10 layers of GO, respectively.

Figure 3(a) shows the calculated BFs (defined as the square of the pulse'rms spectral width at the waveguide output facet divided by the corresponding value at the input) versus GO layer number with input pulse energies of $51.5 \mathrm{pJ}$ and $8.2 \mathrm{pJ}$. There is a maximum BF of 4.14 for the waveguide with 0.4-mm-long, 10 layers of GO, reflecting the trade-off between SPM enhancement and loss increase. By fit the results with SPM theory, we obtained the $n_{2}$ values of GO as a function of GO layer number, as shown in Fig. 3(b). The $n_{2}$ of GO is about 4 orders of magnitude higher than silicon, which agrees reasonably well with our previous FWM experiments [8]. Compared with other 2D materials, $n_{2}$ for GO is lower than graphene but still two orders of magnitude higher than bulk silicon [8-12], which demonstrates the high potential of GO as a new optical material for nonlinear photonic applications with lower TPA than chalcogenides [13], for example. Further, like Si-Ge heterostructures, [14, 15] GO may also offer interesting possibilities for both $2^{\text {nd }}$ and $3^{\text {rd }}$ order nonlinearities due to its complex anisotropic nonlinear optical properties.
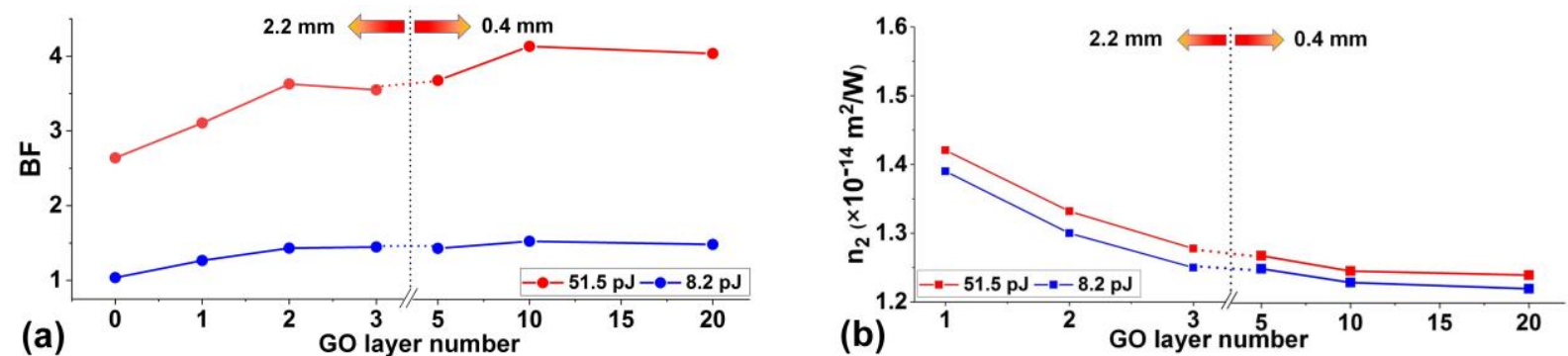

Fig. 3. (a) Calculated BFs versus GO layer number at fixed input pulse energies of $8.2 \mathrm{pJ}$ and $51.5 \mathrm{pJ}$. (b) $n_{2}$ of GO versus layer number at fixed input pulse energies of $8.2 \mathrm{pJ}$ and $51.5 \mathrm{pJ}$.

\section{Conclusion}

We experimentally demonstrate enhanced SPM in SOI nanowires integrated with 2D layered GO films, with a high spectral broadening factor of 4.14 being achieved for a device patterned with 0.4-mm-long, 10 layers of GO.

\section{Reference}

[1] J. Leuthold, C. Koos, and W. Freude, "Nonlinear silicon photonics," Nat. Photon., 4(8), 535, 2010.

[2] J. Wu et.al., "RF photonics: an optical microcombs' perspective," J. Sel. Top. Quantum Electron., 24(4), 1, 2018.

[3] D. Moss et.al., "New CMOS-compatible platforms based on silicon nitride and Hydex for nonlinear optics," Nat. Photon. 7(8), $597,2013$.

[4] H. Bao, et.al, "Laser Cavity-Soliton Micro-Combs", Nature Photonics $\underline{13}$ (6) 384-389 (2019).

[5] T. Gu et.al., "Regenerative oscillation and four-wave mixing in graphene optoelectronics," Nat. Photon., 6(8), 554, 2012.

[6] Y. Yang et.al., "Bottom-up fabrication of graphene on silicon/silica substrate via a facile soft-hard template approach," Sci. Rep., 5, 1, 2015.

[7] X. Zheng et.al., "In situ third-order non-linear responses during laser reduction of graphene oxide thin films towards on-chip non-linear photonic devices," Adv. Mater., 26(17), 2699, 2014. 
[8] Y. Yang et.al., "Enhanced four-wave mixing in waveguides integrated with graphene oxide," APL Photonics, 3(12), 120803, 2018.

[9] J. Wu et.al., "Graphene oxide waveguide and micro-ring resonator polarizers," Laser Photonics Rev., 13(9), 1900056, 2019.

[10] J.Wu et.al., "Enhanced nonlinear FWM in MRRs integrated with layered GO films", Small 16(16), 1906563 (2020).

[11] Y. Zhang et.al, "Enhanced Kerr nonlinearity and nonlinear figure of merit in silicon nanowires integrated with 2D graphene oxide films", ACS Applied Materials and Interfaces $\underline{\mathbf{1 2}}$ (29) 33094-33103 (2020).

[12] D.J. Moss, et.al, "Dispersion in the anisotropy for optical third harmonic generation in Si and Ge", Optics Letters 14 (1), 57-59 (1989).

[13] M. Lamont et.al., "Two Photon Absorption Effects on 2R Optical Regeneration", IEEE Photonics Technology Letters 18 1185 (2006).

[14] M.Sinobad et.al., "Mid-IR octave spanning supercontinuum generation to $8.5 \mu \mathrm{m}$ in Si-Ge waveguides", Optica $\mathbf{5}$ (4), 360-366 (2018).

[15] E.Ghahramani, D.J. Moss, and J.E. Sipe, "Second-harmonic generation in odd-period, strained, (Si)n(Ge)n/Si superlattices and at Si/Ge interfaces", Physical Review Letters, vol. 64, no. 23, pp.2815-2818 (1990). DOI:10.1103/PhysRevLett.64.2815. 